\title{
Reference systems and the perception of tactual and haptic orientation
}

\author{
JOHN J. RIESER and HERBERT L. PICK, JR. \\ University of Minnesota, Minneapolis, Minnesota 55455
}

\begin{abstract}
Adult subjects learned to identify bars differing in orientation. The bars were presented either tactually or haptically. In the first experiment, learning was followed by a transfer test with body posture changed by $90 \mathrm{deg}$. That is, if subjects originally learned with body upright, the transfer test was carried out with body reclined. Results of the transfer test indicated original learning of the tactually presented bars was done with respect to a body reference system and original learning of the haptically presented bars was done with respect to an environmental or gravity based reference system. In the second experiment, learning was followed by a transfer test using both a change of body posture and a change of stimulus modality from tactual to haptic or vice versa. Performance in this transfer test is interpreted in terms of a conceptual mediation hypothesis.
\end{abstract}

The identification of stimuli differing only in orientation is ambiguous, since judgment of their orientation itself depends on the frame of reference used. For example, whether $W$ is perceived as the 13 th or 23 rd letter of the alphabet depends on the frame of reference for its perception. What determines the frames of reference used for a particular judgment and how they are used? The series of experiments reported here was designed to examine our use of reference systems when making tactual or haptic perceptual identifications of objects differing only in their orientation in the frontoparallel plane. Subjects with closed eyes were tested with objects which they actively grasped by hand (haptic) or had passively pressed onto the forehead (tactual). At least two reference systems are potentially relevant: for one, up-down is defined in relation to gravity, and for the other, up-down is defined in relation to observer's body.

Directions determined by these two reference systems are not always the same. For example, consider a blindfolded subject who is asked to learn an arbitrary name, e.g., "v," for an objectively vertical bar which he feels with his hand and another arbitrary name, e.g., "h," for an objectively horizontal bar. Now, suppose the blind folded subject is asked to identify these same bars while lying on his side. Will he respond in terms of an objective. gravity-based reference system and call the same bars "v" and "h," or will he respond in terms of an egocentric. body-based reference system and.called the original vertical bar " $h$ " and the original horizontal bar " $v$ "?

This research was supported by a Program Project Grant HD-03082 from the National Institutes of Health to the Institute of Child Development of the University of Minnesota and by the Center for Research in Human Learning of the University of Minnesota.
Using four orientations and a 45-deg change in head tilt. Attneave and Olson (1967) reported that most subjects use a gravity-based reference system to identify visual bars when there is no visual environmental trame of reference. The generality of this result was extended by Rock (1973) to include a great number of visual stimuli and many head positions.

The use of a gravity-based reference system is surprising in some respects. After a change in head position. the retinal image of a stationary object is rotated with respect to the retina. Correct identitication of that rotated image implies that the process of recognition somehow takes into account the change in body position. The anatomical basis for such a process is not known. However, evidence like the very fast recognition times reported by Attneave and Olson (1967) and Rock (1973) suggests that the process is automatic and not thoughtful. Logically, correct identification would seem to require the interaction of visual sensory information specifying the orientation of the image on the retina with information for the orientation of the retina (or head) with respect to the world.

In general, two types of sensory information can act to signal a person's body position with respect to gravity: ordinarily, the visual field including a distinctive floor and ceiling is congruent with gravity up-down. while proprioceptive stimulation including the vestibular and kinesthetic information may directly signal the direction of gravity. When visual cues for body orientation are eliminated, a gravity-based reference system will continue to be used with many visual stimuli (Rock, 1973). Thus, convergence of proprioceptive with visual (retinal orientation) information seems sufficient for use of a gravity-based reference system.

In the present study, interest is focused on the identification of stimuli in the frontoparallel plane 
Table 1

Experiment I: Training and Transfer Data

\begin{tabular}{|c|c|c|c|c|c|}
\hline $\begin{array}{l}\text { Training } \\
\text { Condition }\end{array}$ & & $\mathrm{T} \uparrow$ & $\mathrm{T} \rightarrow$ & $\mathrm{H} \uparrow$ & $\mathrm{H} \rightarrow$ \\
\hline $\begin{array}{l}\text { Transfer } \\
\text { Condition }\end{array}$ & & $\mathrm{T} \rightarrow$ & $\mathrm{T} \uparrow$ & $\mathrm{H} \rightarrow$ & $\mathrm{H} \uparrow$ \\
\hline Learning & $\begin{array}{l}\text { Trials to } \\
\text { Criteria }\end{array}$ & 80.0 & 92.8 & 82.7 & 80.0 \\
\hline Transfer & $\begin{array}{l}\text { Gravity } \\
\text { Ref System }\end{array}$ & 0 & 0 & 12.0 & 15.5 \\
\hline \multirow[t]{2}{*}{$\begin{array}{l}\text { Mean Number } \\
\text { Trials Out of } 16\end{array}$} & $\begin{array}{l}\text { Body Ref } \\
\text { System }\end{array}$ & 14.8 & 15.0 & 1.8 & .0 \\
\hline & Errors & 1.2 & 1.0 & 2.2 & .3 \\
\hline
\end{tabular}

Note-Modality $(T=$ tactual. $H=$ haptic $) ;$ body orientation $\uparrow \uparrow$ upright,$\rightarrow=$ on-side $)$.

that differ only in their orientation with respect to gravity. Information for their orientation is provided only haptically or tactually. Thus, for some types of judgments, there might be convergence of proprioceptive information about direction of gravity with haptic or tactual information about orientation with respect to the body. Two main experiments are reported. In the first, the major question is what frames of reference are used in the identification of haptic and tactual stimuli differing only in orientation. Results indicate that identification of tactual stimuli differing in orientation is made in terms of a body reference system and identification of haptic stimuli is made in terms of an objective or gravity reference system. This difference was not due to a difference in active as compared to passive stimulus exposure. In the second experiment, a crossmodal procedure was used to suggest answers to questions about how and when orientation information is encoded.

\section{EXPERIMENT I}

\section{Method}

In this experiment, modeled after Attneave and Olson (1967), subjects were trained to identify either haptic or tactual stimuli differing in up-down orientation. They were then tested for recognition with body orientation changed by $90 \mathrm{deg}$. The purpose was to determine whether subjects identified stimuli in terms of objective or body reference systems.

Haptic stimuli were presented by means of a steel bar, 10 in. long, $1 / 4$ in. diam., Mounted in the center of the bar was a circular, finger-sized starting point slightly raised above the bar's surface. The bar could be rotated in the subject's frontal plane to each of four fixed orientations: vertical, horizontal, and the two $45-\mathrm{deg}$ diagonals. The bar was positioned about $1 \mathrm{ft}$ from the subject centered near the midline at chest level.

Tactual stimuli were presented by means of a piece of wax, $21 / 2$ in. long. Spaced at equal intervals were five $1 / 8$-in.-diam nail heads extending above the bar's surface. The same four orientations described for the haptic bar were also used tactually. Bar orientation was adjusted manually by the experimenter, who was guided by a set of external reference lines adjacent to the subject's head. Although not closely controlled, the center of the bar for each of the four orientations was at a central location on the forehead.
The wax bar was curved for each subject. so that on each presentation all the nail heads made contact with the skin.

Two body postures were used: standing upright and lying on a side. For each posture. subjects were instructed to keep head and body aligned.

Subjects were 24 University of Minnesota men and women undergraduates. They were assigned randomly to one of four conditions. Two groups received haptic stimuli and two tactual. One haptic group and one tactual group were trained standing upright. The other two groups were trained while lying on their sides. Subjects were instructed to keep their eyes closed, and at no time did they see the stimulus bars.

There were two phases in the experiment: training and transfer. During training. the subject stood upright or lay on his side. His task was to learn a letter name $(a, c, o$, or $s)$ for each of the four stimulus orientations. On each trial a stimulus was presented, the subject responded with the name and received error feedback and general encouragement from the experimenter. Presentation of haptic bars involved relatively active participation by the subject. On each trial, the subject's index finger was placed on the bar's starting point. At a verbal starting signal, he quickly grasped the bar. Presentation of tactual bars was relatively passive. On each trial, a verbal warning signal was followed by the impression of the bar on the forehead.

In all conditions, a subject was introduced to the four stimulus orientations and corresponding letter names with two cycles through the four stimuli. After this introduction, subjects were trained to a criterion. Random series consisting of 16 trials each were constructed; each series consisted of four trials with each of the four orientations. After the first two series were completed, the subject was asked to respond as quickly as possible. Criterion learning was defined as the first error-free series following the third series. Intertrial intervals were not controlled. but averaged 3 sec.

The transfer phase involving a 90-deg change in body tilt followed training. At this time, subjects were asked to simply identify the stimuli as quickly as possible, offering the first plausible letter name that occurred to them. In the rare cases when a subject interrupted the transfer phase with a question, his request was noted and he was promised a full explanation following testing. The transfer test consisted of one random series of 16 trials. No error feedback was given during transfer.

At the conclusion of the experiment, subjects were interviewed to shed light on three questions: (1) Did the problem of two possible reference systems occur to them? (2) Were they aware of thinking about bar orientations with words during the session? (3) Were they aware of visualizing the bars during the session?

\section{Results}

Subjects generally reached training criterion after about 80 trials (the few subjects failing to reach criterion within 128 trials were discontinued at that time). A three-category code was developed for transfer responses. so that responses within a condition could be grouped across the four stimulus orientations in a form relevant to questions about the frame of reference. The responses could: (a) indicate the use of a gravity or objective reference system, (b) indicate the use of a body or egocentric reference system, or (c) be inconsistent with either of these, and for present purposes are classified as errors. A gravity reference system would be implied, for example, if an objectively vertical bar presented haptically was labeled " $a$ " while the subject was standing upright and if the same response was made to the objectively vertical bar in the transfer test with the subject lying on his side. A body reference system would be 
implied, on the other hand, if that objectively vertical bar was labeled "a" while the subject was upright but was labeled with the name of the horizontal stimulus when the subject was tested for transfer on his wide. If the subject during transfer gave a name to the objectively vertical bar appropriate to one of the diagonals, that would be considered an error.

The results are presented in Table 1 . There were no significant differences among the groups in initial learning. The data indicate quite clearly that the tactual subjects respond on the basis of a body reference system and the haptic subjects on the basis of a gravity reference system. That result is not only true on the average but characterizes all 12 tactual subjects and 11 out of 12 haptic subjects. One subject who was trained in the haptic upright condition and transferred to the haptic on-side condition made 11 out of 16 transfer responses in terms of a body reference, with the other 5 responses being errors.

\section{Discussion}

The haptic judgments presumably involve information from receptors in the elbow and shoulder joints converging with information about gravity, e.g., from the vestibular system. The elbow and shoulder joint information may be available for use unintegrated with information about gravity and hence account for the one haptic subject who responded in terms of a body reference system. Furthermore, if indeed the joint information is available for use directly, one would predict that subjects might voluntarily shift back and forth between a body or an objective reference system in learning haptic orientations. It seems quite reasonable that there would be an initial preference to use a gravity reference system integrated with joint information. For example, the numerous postural reflexes very elegantly coordinate joint information with information about body orientation with respect to gravity.

On the other hand, there is no analogous advantage for the convergence of tactual receptors with body position information. In fact, one wants to know sources of skin stimulation, e.g., irritation, in relation specifically to a body reference system. Consistent with this line of reasoning is recent electrophysiological data on squirrel monkeys. Schwartz and Fredrickson (1971) recorded from single units in the parietal lobe, finding many units responsive to vestibular inputs. Many of these units were also responsive to joint receptor stimulation, whereas none of these units responded to light pressure stimulation (analogous to tactual stimulation in the present study).

Another possible reason for the strong preference for a body reference system with the tactual stimuli is the use of the forehead as the stimulated surface. The hairline, eyes, and nose framing the forehead could constitute biasing cue stimuli for use of a body reference system. Four additional subjects were run in the tactual conditions, but with stimuli impressed onto the abdomen surface. These subjects also all responded in terms of a body reference system.

The haptic and tactual conditions of Experiment I differed on still another dimension sometimes found to be psychologically important. Perception of the haptic stimuli was a rather active task, whereas perception of the tactual stimuli was accomplished very passively. It was difficult to devise ap active tactual task, but six additional subjects were tested in a passive haptic task. The stimuli and procedures were identical to those described for Experiment I for haptic stimuli. However, on each trial, subjects were asked to keep arms and hands as relaxed as possible and the experimenter placed the subject's hand on the stimulus bar on each trial. All six subjects used an objective reference system $(89 \%$ of the responses were consistent with a gravity reference system). Thus, the difference between haptic and tactual perception in Experiment I cannot be easily accounted for by the different activity level of the two tasks.

\section{EXPERIMENT II}

In the first experiment, it was found that the tactual and haptic modalities characteristically elicited use of different reference systems to identify stimulus orientation. Thus, the task used here produces systematically different responses depending on the sensory modality tested. Suppose, after learning to identify a set of stimuli as in Experiment I, subjects were confronted with a change in sense modality as well as a change in body orientation. What reference systems would be used in identification of these stimuli? Experiment 11 was designed to investigate this question. Perhaps the simplest hypothesis would be that the modality of initial training would determine the reference system used. Thus, if a subject learned to identify tactual stimuli while standing upright, when tested haptically on his side he would respond in terms of a body reference system (identifying an objectively vertical stimulus with the same name he used for a horizontal stimulus during training).

\section{Method}

Twenty-four new subjects participated, six in each of four cross-modal conditions. In such a cross-modal study, subjects could be trained with haptic or tactual stimuli with body orientation upright or on the side. Each of these four training conditions unambiguously determined a transfer condition in which modality and body orientation was changed. For example, an upright tactual training condition could only be followed by an on-side haptic transfer condition.

The training and transfer procedures were the same as those used in Experiment I, except for the change in sense modality of stimuli between transfer and training. 
Table 2

Experiment II: Training and Transfer Data

\begin{tabular}{|c|c|c|c|c|c|}
\hline $\begin{array}{l}\text { Training } \\
\text { Condition }\end{array}$ & & $T \uparrow$ & $\mathbf{T} \rightarrow$ & $\mathrm{H} \uparrow$ & $\mathbf{H} \rightarrow$ \\
\hline $\begin{array}{l}\text { Transfer } \\
\text { Condition }\end{array}$ & & $\mathrm{H} \rightarrow$ & $H \uparrow$ & $\mathrm{T} \rightarrow$ & $\mathrm{T} \uparrow$ \\
\hline Learning & $\begin{array}{l}\text { Trials to } \\
\text { Criterion }\end{array}$ & 82.3 & 74.7 & 77.3 & 77.3 \\
\hline Transfer & $\begin{array}{l}\text { Gravity } \\
\text { Ref System }\end{array}$ & 11.8 & .7 & 1.5 & 14.8 \\
\hline $\begin{array}{l}\text { Mean Number } \\
\text { Trials Out of } 16\end{array}$ & $\begin{array}{l}\text { Body Ref } \\
\text { System }\end{array}$ & 2.7 & 15.2 & 12.7 & .2 \\
\hline & Iirrors & 1.5 & .2 & 1.8 & 1.0 \\
\hline
\end{tabular}

Nole-Modality $(T=$ tactual, $\boldsymbol{H}=$ haptic $)$ body orientation $(1=$ upright,$\rightarrow=$ on-side $)$.

\section{Results and Discussion}

The results are shown in Table 2. The tactual upright training and haptic on-side training conditions yielded responses in terms of a gravity reference system, while the tactual-on-side training and haptic upright training conditions yielded responses in terms of a body reference system: Thus, the simple hypothesis that the preferred reference system of the training modality would be the one used in the cross-modal transfer condition was not supported. Nor was an alternative hypothesis supported that the transfer modality determined the reference system used. As noted from Table 2, the haptic on-side and the tactual upright transfer conditions seemed to involve the gravity reference system, while the haptic upright and tactual on-side transfer conditions involved a body reference system.

Perhaps the results are more reasonably understood in terms of a mediation model. Suppose that during training a person learns to label particular phenomenal directions rather than the specitic stimuli. For example, in the tactual on-side training condition, the subject might be given the task of associating the name "a" with an objectively up-right stimulus impressed on his forehead. That stimulus is, of course, running left-right on his body, and from Experiment $I$ we assume that he is using a body reference system. We hypothesize he in fact is learning to associate the name "a" with the phenomenal horizontal or side to side. In the cross-modal transfer conditions, the subject is standing upright and feeling a horizontal bar. He, of course, experiences this as horizontal and provides the appropriate label, "a." By our classification, he responds in terms of a body reference system. Take another example, In the haptic upright training condition, the subject feels a vertical bar and learns to associate, say, "s" with the phenomenal experience of vertical or up-down. Now, in the tactual on-side transter condition, an objectively horizontal bar is impressed on his forehead and is oriented up-down with respect to his body. Again from Experiment I, we infer that the subject experiences this in terms of a body reference system as up-down and that he applies the label "s" he has learned for that phenomenal orientation; that is, he responds with a label appropriate for a body reference system. This fornulation lits the other results of Experiment II in a similar manner.

The formulation also fits two other supplementary cross-model conditions run as part of Experiment II. These two conditions, procedurally similar to the four previously described were: (a) tactual on-side training to haptic on-side transfer, and (b) haptic on-side training to tactual on-side transfer. In (a), six subjects lying on their sides learned to associate the set of labels to tactual stimuli. When they were transferred, still lying on their sides, to haptic stimuli, they shifted their labeling on 15 out of 16 trials as if from a body to gravity reference system. Similarly (but not as dramatically), in condition (b), six subjects shifted their labeling as if from a gravity to a body-reference system. In that condition, an average of 7.2 out of 16 transfer trials showed such a shift. An average of 4.3 responses continued to be consistent with a gravity reference system, and 4.3 trials were inconsistent and classified as errors. These shifts again make sense in terms of a conceptual mediation model. Consider the condition (a), tactual on-side training to haptic on-side transfer. In training, the subject learns to associate the label "a" with an objectively vertical pressure on his forehead. But from Experiment I, we infer he is experiencing this as horizontal. Now, in transfer, when he is presented with a haptic objectively horizontal bar, he experiences that as horizontal and calls it "a."

Thus, it appears that the cross-modal matching can be parsimoniously understood in terms of a mediation model. It is not clear at this point whether the mediation is conceptual or verbal, or both. However, at least two factors argue against the primacy of verbal mediation. First, in the postexperiment interviews, fewer than one-third of the subjects reported consciously using words to describe perceived directions. Some subjects reported responding simply on the basis of the "feel" of the stimulus bars. Many of the subjects who did report using verbal labels became confused when asked to state verbal codes for either of the two diagonal bars. Second, the use of verbal coding, e.g., horizontal, vertical, etc., would seem logically to require prior conceptual or phenomenal identification in order to permit use of the appropriate verbal mediators.

In summary, the results of Experiment I imply that tactual identifications of stimuli differing in orientation are made using a body reference system and haptic identilications are made using a gravity or objective reference system. Such modality-reference 
system preferences or biases seem intuitively reasonable in terms of other modality-specific tasks (cf. discussion of Experiment I). On the basis of Experiment 1I, it may be inferred that th stimulus identifications are associations between the experimenter's arbitrary labels and the phenomenal or conceptual orientation of the stimuli. Then, if the subject is tested for recognition in a different modality, the orientation experienced on the basis of that modality's preferred reference system determines the subject's response.

\section{REFERENCES}

Attneave, F., \& Olson, R. K. Discriminability of stimuli varying in physical and retinal orientation. Journal of Experimental Psychology, 1967, 74, 149-157.
Attneave, F., \& Reid, K. W. Voluntary control of frame of reference and slope equivalence under head rotation. Journal of Experimental Psychology, 1968, 78, 153-159.

FREIDEs, D. Human information processing and sensory modality: Cross-modal functions, information complexity, memory and deficit. Psychological Bulletin, 1974, 81, 284-310.

HeLD, R., \& Hein, A. V. Adaptation of disarranged eye-hand coordination contingent on re-afferent stimulation. Perceptual and Motor Skills, 1958, 8, 87-90.

Rock, I. Form and orientation. New York: Academic, 1973.

SChwarz, D. W. F., \& Fredrickson, J. M. Rhesus monkey vestibular cortex: A bimodal primary projection field. Science, $1971,172,280-281$.

(Received for publication September 22, 1975; revision received November 20,1975 .) 